\title{
A VARIATIONAL METHOD FOR MULTISTAGE LAUNCH VEHICLE OPTIMIZATION
}

by Fred Teren and Omer F. Spurlock

Lewis Research Center

Cleveland, Ohio

TECHNICAL PAPER proposed for presentation at

Third Aerospace Sciences Meeting of the American Institute of Aeronautics and Astronautics New York, New York, January 24-26, 1966

NATIONAL AERONAUTICS AND SPACE ADMINISTRATION 



\section{A VARIATIONAL METHOD FOR MUTISTACE LAUNCH VEHICLE OPTIMIZATION}

by Fred Teren and Omer F. Spurlock

Lewis Research Center

National Aeronautics and Space Administration

Cleveland, Ohio

\section{ABSTRACT}

14770

rine methods of the calculus of variations are used to maximize payload capabizity for waltistage launch vehicles. The method of solution uses the Iagrange misipiers to determine the opimun thrust direction profile, as well as to construct partial derivatives of payload with respect to the stage rorellant loadings and a booster steering parameter. These derivatives are : sed to terminate the stages and/or as terninal equations to be satisfied. Maximum payload can thus be achieved with a single solution, rather than with a f'aniiy of paranetric resuits. Constant thrust ard specific-impulse operation is assuned for each upper stage (booster thmust ard specific impulse vany with atrospheric pressure), and structure weight can be either fixed or a linear function of the stage propellant loadirg. Two-dimensional flight ir a central, inverse-s zuare gravitational fiela is assimed.

Iumerical resiuts are presented for two- and three-stage launch vehicles flown to ciralar orbit and Earth escape, respectively. Parametric resilts ane preserted and compered with the overall optimin solution obtained by use ot the variational technique.

\section{INITRODUUTION}

A problem that frecuently arises in trajectory optimization studies is that of determining the maximum payload capability of a multistage launch rehicle flown to a prescribed set of burnout conditions. If all vehicle paraneters are specified, the problem reduces to that of finding the optimum 
steering profile. In many cases, however, not all these parameters are specified, and those left unspecified can be varled to maximize payload.

A typical situation that occurs in the design of future launch vehicles is one in which the stage thrust levels and propellant flow rates (and, for practicality, the gross launch weight) are specified, but some or all the stage propellant loadings are left unspecified. The unspecified propellant loadings generally can be varied to achieve maximum payload capability for the vehicle.

An additional optimizing parameter frequently is available in the booster steering program. Since the booster stage operates in the atmosphere, the booster thrust direction profile is shaped to minimize aerodynamic heating and loads and is not available for complete optimization. A single degree of freedom remains, however, corresponding to the magnitude of a short pitchover phase following the initial vertical rise. This degree of freedom, sometimes called the booster kick angle, determines the amount of trajectory lofting during boost phase. Since the upper stages operate essentially under vacuum conditions, the steering program for these stages is available for complete optimization.

Many authors (e.g., refs. I to 7) have treated the problem of optimizing the stage propellant loadings of multistage vehicles. None of these authors, however, has attempted to optimize the steering program for these vehicles. Others (e.g., refs. 8 to 10 ) have used the calculus of variations to optimize the steering program for various rocket vehicles. In particular, reference 11 treats the problem of optimizing the steering program of a multistage launch vehicle. Reference 11, however, does not consider the problem of optimizing the stage propellant loadings or booster kick angle.

Recently, Mason, Dickerson, and Smith (ref. 12) have considered the problem of simultaneously optimizing the steering program and the stage propellant 
loadings of a miltistage launch vehicle. These authors followed the approach of Denbow ( problem.

The present report was written concurrently with reference 12 and presents a riethod which allows the propellant loadings, booster kick angle, and upperstage steering program to be simultaneously optimized. The variational approach is somewhat different from that used in reference 12. By following the method of reference 15, the maximizing functional is written as the sum of the final rayload and a constraint integral for each of the upper stages. The resulting boundary equations suppiy partial derivatives of payload with respect to the unsperified parameters. These derivatives are then used, along with the required burnout conditions, as terminal equations to be satisfied. The analysis does not require that all the stage propellant loadings (or booster kick angle) be optimized. Eciations are developed for optimizirg payload with respect to a:y combination of unspecified parameters.

The variational equations for optimizing vehicle parameters have been incorporated into a digital computer program used previously at Lewis for parametrio zaunct-vehicle studies. Some of the procedures used to obtain numerical. results with this program are discussed in reference 16. Numerical results are preserted for two- and three-stage launch vehicles flown to circular orbit and Earth escape, respectively, to demonstrate the feasibility of the variational approach. Parametric results are presented showing the variation of payload with propellant loadings and booster kick angle. The resulting paylond envelopes are then compared with the overall optimum points generated aineatly by means of the variational technique in order to verify the equations. ANALYSIS

The problem to be solved is to determine the maximum payload capability of an N-stage launch vehicle flown to a specitied set of burnout conditions. 
The analysis admits atmospheric effects during booster phase but assumes vacuum . operation for alI other stages. Because of these atmospheric effects, the booster steering program is assumed completely specified (e.g., zero angle of attack), except for the booster kick angle. The upper-stage steering program, however, is unconstrained and is determined to maximize payload. The calculus of variations is used for this purpose.

Each of the upper stages is assumed to operate at a fixed (constant) value of thrust and propellant flow rate. These values may be zero, so that coast phases are admitted. The structure mass for each stage is assumed to be a linear function of the stage propeliart loading, defined by

$$
\mathrm{m}_{\mathrm{S}}=\mathrm{m}_{\mathrm{H}}+\mathrm{km} \mathrm{m}_{\mathrm{p}}
$$

where $m_{S}$ is the total structure mass, $m_{H}$ is the fixed mass, $m_{p}$ is the stage propellant mass, and $\mathrm{k}$ is the propellant sensitive mass fraction. (All symbols are defined in appendix A.)

In addition to the variational trajectory, provision is also made for adding an additional velocity increment $\Delta v_{I}$ after the desired orbit conditions are achieved. This velocity increment is achieved by use of the final stage for propulsion. The amount of propellant required for this maneuver is calculated by use of the standard impulsive velocity equations.

\section{Variational Problem}

Since the booster steering program is not subject to complete optimization, the booster stage is not treated in the following Euler-Lagrange equations. The booster degrees of freedom (propellant loading and kick angle) are included by allowing variations in the position and velocity at second-stage ignition. The associated equations, along with the equations for optimizing upper-stage propellant loadings, are treated in the boundary equations resulting from the variational analysis.

The variational problem to be solved is that of finding the upper-stage 
thrust program which maximizes the payload capability of an $N$-stage launch vehicle for given boundary conditions. This problem can be formulated as a generalized Bolza problem. By following the treatment in reference 15, the Iunctional to be minimized can be written as

$$
J=g+\sum_{i=2}^{\mathbb{N}} \int_{t_{i-1}}^{t_{i}} F_{i} d t
$$

where $g$ is a function of initial and final conditions to minimized and $F_{i}$ consists of a set of constraints to be applied to each of the upper stages, added together with the aid of Lagrange multipliers. For this problem, the rayload is to be maximized, so that

$$
g=-m_{P \bar{L}}
$$

Fihe constraint equations are

$$
\begin{aligned}
& f_{1 i}=\dot{u}+\frac{\mu}{r^{2}}-\omega^{2} r-\frac{T_{i}}{m} \sin \psi=0 \\
& f_{2 i}=r \dot{\omega}+2 u \omega-\frac{T_{i}}{m} \cos \psi=0 \\
& f_{3 i}=\dot{r}-u=0 \\
& f_{4 i}=\dot{\varphi}-\omega=0 \\
& f_{5 i}=\dot{r}+\beta_{i}=0
\end{aligned}
$$

appisatie on the interval

$t_{i-1} \leq t \leq t_{1}$

for $i=2,3, \ldots, \ldots, \mathbb{N}$

Eqiations (3a) to (3d) are the two-body equations of motion written in twodimersional polan coordinates with ar inverse square force field acting. The trust direction $\psi$ and the state variables are defined in figure 1 . Equation ( $3 e$ ) defines the propellant flow rate.

Equations (3) are combined to give

$$
F_{i}=\sum_{j=1}^{5} \lambda_{j i} f_{j i} \quad i=2, \ldots ., N
$$


where $\lambda_{j i}$ are undetermined Lagrange multipliers, which are functions of time since the constraint equations must be satisfied at all points of the trajectory.

\section{Euler-Lagrange Equations}

As shown in reference 17 , a necessary condition for $g$ to be minimized is that the Euler-Lagrange equations be satisfied. The Euler-Lagrange equations are

$$
\frac{d}{d t}\left(\frac{\partial F_{i}}{\partial t_{j}}\right)=\frac{\partial F_{i}}{\partial x_{j}} \quad i=2, \ldots ., N ; j=1, \ldots, 6
$$

where $x_{j}$ are the problem variables

$$
\left.\begin{array}{l}
x_{1}(t)=u \\
x_{2}(t)=\omega \\
x_{3}(t)=r \\
x_{4}(t)=\varphi \\
x_{5}(t)=m \\
x_{6}(t)=\psi
\end{array}\right\}
$$

The Euler-Lagrange equations for the present problem can be written explicitly by use of equations (3) and (4):

$$
\begin{aligned}
& \dot{\lambda}_{1}=2 \omega \lambda_{2}-\lambda_{3} \\
& \dot{\lambda}_{2}=-2 \omega \lambda_{1}+\frac{u}{r} \lambda_{2}-\frac{\lambda_{4}}{r} \\
& \dot{\lambda}_{3}=-\left(\frac{2 \mu}{r^{3}}+\dot{\omega}^{2}\right) \lambda_{1}+\dot{\omega} \lambda_{2} \\
& \dot{\lambda}_{4}=0 \\
& \dot{\lambda}_{5}=\frac{T}{m^{2}}\left(\lambda_{1} \sin \psi+\lambda_{2} \cos \psi\right) \\
& \left(\lambda_{1} \cos \psi-\lambda_{2} \sin \psi\right) \frac{T}{m}=0
\end{aligned}
$$

Equations (7) (and subsequent equations) apply separately to each of the upper stages. The subscript $i$ has been omitted for simplicity.

Equation ( $7 f$ ) determines the thrust direction (for $T \neq 0$ ): 


$$
\begin{aligned}
& \tan \psi=\frac{\lambda_{1}}{\lambda_{2}} \\
& \sin \psi=\frac{\lambda_{1}}{\sqrt{\lambda_{1}^{2}+\lambda_{2}^{2}}} \\
& \cos \psi=\frac{\lambda_{2}}{\sqrt{\lambda_{1}^{2}+\lambda_{2}^{2}}}
\end{aligned}
$$

The choice of sign in equations ( $8 b)$ and $(8 c)$ has been determined by use of the Weierstrass E-test (ref. 18).

Equations ( $7 a$ ) to (7d) must be integrated, along with the equations of motion to determine the thrust direction and the optimum trajectory. It is shown later that equation (7e) need not be Integrated. Equation (7d) is easily integrated to give

$$
\lambda_{4}=\text { Constant }
$$

First Integral

Since the function $F$ does not contain the independent variable (time) explicitly, a first integral to the Euler-Iagrange equations exists (for each stage), which can be stated as (ref. 17)

$$
F-\sum_{j=1}^{6} \frac{\partial F}{\partial \mathrm{x}_{j}} \dot{x}_{j}=\mathrm{C}
$$

Equation (10) can be written explicitly by use of equations (3) and (4):

$$
c-\left(\frac{\mu}{r^{2}}-\omega^{2} r\right) \lambda_{1}-2 u \omega \lambda_{2}+u \lambda_{3}+\omega \lambda_{4}-\beta \lambda_{5}+\frac{T}{m}\left(\lambda_{1} \sin \psi+\lambda_{2} \cos \psi\right)=0
$$

Equation (1I) is used later in the analysis to eliminate both $C$ and $\lambda_{5}$ from the boundary equations.

\section{Weierstrass-Erdmann Corner Condition}

The boundary conditions on the Lagrange multipliers at staging points can be derived with the aid of the Weierstrass-Frdmann corner condition (ref. 17). This condition states that $\frac{\partial F}{\partial x_{j}}(j=1, ., ., 6)$ must be continuous 
at such corners. For the present problem, this condition implies that all the multipliers are continuous at staging points, hence continuous throughout the trajectory.

\section{Transversality Equation}

The relation between changes in boundary conditions and changes in $J$ is expressed by the general transversality equation (ref. 17). For this problem, the transversality equation can be written

$$
\mathrm{d} J=\sum_{i=2}^{N}\left[\left(F_{i}-\sum_{j=1}^{6} \dot{x}_{j} \frac{\partial F_{i}}{\partial \dot{x}_{j}}\right) d t+\sum_{j=1}^{6} \frac{\partial F}{\partial \dot{x}_{j}} d x_{j}\right]_{t_{i-1}}^{t_{i}}+d g
$$

This equation can be written explicitly by using the definition of $F$ and the first integral

$$
\mathrm{d} J=\sum_{1=2}^{\mathrm{N}}\left(\mathrm{c}_{i} \cdot \mathrm{dt}+\lambda_{1} d u+r \lambda_{2} d \omega+\lambda_{3} d r+\lambda_{4} d \varphi+\lambda_{5} d \mathrm{~m}_{\mathrm{i}}\right)_{t_{i-1}}^{t_{i}}+\mathrm{dg}
$$

The subscript $i$ has been used with $C$ and $d m$ since these variables may be discontinuous at staging points.

\section{Boundary Equations}

If some of the problem variables (state conditions or control variables) are not specifled, values should be chosen which minimize $J$ (or, equivalently, maximize payload).

According to reference 17, minimizing $J$ is accomplished by setting dJ equal to zero. Equation (13) has the form

$$
d J=\sum_{j=1}^{m} G_{j} d x_{j}=0
$$

If the $m$ problem variables $x_{j}$ are all independent, $d J$ wil vanish if, and only if, each term on the right side of equation (14) is independently set equal to zero. For specified variables $x_{j}$, the allowable variation $\mathrm{dx}_{j}$ is zero; for unspecified $x_{j}$, the coefficient $G_{j}$ must be set equal to zero. Equa- 
tion (14) can thus be interpreted as a total differential of $\mathrm{J}$, and

$$
G_{j}=\frac{\partial J}{\partial \mathrm{x}_{j}}
$$

Equation (13) is not suitable for this interpretation, since the variables are not all independent. In the following section the dependent variables are eliminated by expressing the dependence explicitly.

Consider first the terms in equation (13) involving the variations of the state variables.

$$
\begin{aligned}
\sum_{1=2}^{\mathbb{N}}\left(\lambda_{1} d u+r \lambda_{2} d \omega+\lambda_{3} d r+\lambda_{4} d \varphi\right)_{t_{i-1}}^{t_{i}}=-\sum_{1=2}^{N-I}\left[\left(\lambda_{1} d u\right)^{+}-\left(\lambda_{1} d u\right)^{-}+\left(r \lambda_{2} d \omega\right)^{+}\right. \\
\left.\quad-\left(r \lambda_{2} d \omega\right)^{-}+\left(\lambda_{3} d r\right)^{+}-\left(\lambda_{3} d r\right)^{-}+\left(\lambda_{4} d \varphi\right)^{+}-\left(\lambda_{4} d \varphi\right)^{-}\right]_{t=t_{i}} \\
\quad+\left(\lambda_{1} d u+r \lambda_{2} d \omega+\lambda_{3} d r+\lambda_{4} d \varphi\right)_{t=t_{\bar{N}}}-\left(\lambda_{1} d u+r \lambda_{2} d \omega+\lambda_{3} d r+\lambda_{4} d \varphi\right)_{t=t_{1}^{+}}
\end{aligned}
$$

where the superscripts - and + refer to conditions before and after staging, respectively. Since the state variables and Lagrarge multipliers are continuous throughout the trajectory, the summation on the right side of equation (15) is identically zero. At $t=t_{1}$, the variations in state conditions are due to the allowable variations in booster burning time and kick angle.

$$
\left(d x_{j}\right)_{t=t_{I}^{+}}=\frac{\partial x_{j}}{\partial \tau_{I}} d \tau_{I}+\frac{\partial x_{j}}{\partial \alpha} d \alpha \quad j=1, \ldots, 4
$$

where $\alpha$ is the booster kick angle and $\tau_{1}$ is the booster burning time. (In general, $\tau_{i}$ is the burring time of stage 1 , that is, $\left.\tau_{i}=t_{i}-t_{i-1}\right)$. For generality, the state variations at $t=t_{N}^{-}$are expressed in terms of a set of generalized (independent) state variables, $\eta_{\mathrm{k}}, \mathrm{k}=1, . . .4$, so that

$$
\mathrm{dx}_{\mathrm{j}}=\sum_{\mathrm{k}=1}^{4} \frac{\partial \mathrm{x}_{\mathrm{j}}}{\partial \eta_{\mathrm{k}}} \mathrm{d} \eta_{\mathrm{k}} \quad \mathrm{j}=1, \ldots, 4
$$

Combining equations (15) to (17) yields 


$$
\begin{aligned}
& \sum_{1=2}^{\mathbb{N}}\left(\lambda_{1} d u+r \lambda_{2} \partial \omega+\lambda_{3} d r+\lambda_{4} d \varphi\right)_{t_{i-1}}^{t_{i}}=\sum_{k=1}^{4}\left(\lambda_{1} \frac{\partial u}{\partial \eta_{k}}+r \lambda_{2} \frac{\partial \omega}{\partial \eta_{k}}+\lambda_{3} \frac{\partial r}{\partial \eta_{k}}+\lambda_{4} \frac{\partial \varphi}{\partial \eta_{k}}\right)_{t=t_{N}} d \eta_{k} \\
& -\left(\lambda_{1} \frac{\partial u}{\partial \alpha}+r \lambda_{2} \frac{\partial \omega}{\partial \alpha}+\lambda_{3} \frac{\partial r}{\partial \alpha}+\lambda_{4} \frac{\partial \varphi}{\partial \alpha}\right)_{t=t_{1}^{+}} d \alpha-\left(\lambda_{1} \frac{\partial u}{\partial \tau_{1}}+r \lambda_{2} \frac{\partial \omega}{\partial \tau_{1}}+\lambda_{3} \frac{\partial r}{\partial \tau_{1}}+\lambda_{4} \frac{\partial \varphi}{\partial \tau_{1}}\right)_{t=t_{1}^{+}} d \tau_{1}
\end{aligned}
$$

Inasmuch as a suitable form for the state variation terms in equation (13) has been obtained, the mass and payload variation terms are now considered. Since the propellant flow rate is constant for each stage, the variables $m_{i}$ can be expressed as functions of the burning times of the stages. Specifically,

$$
\left.\begin{array}{l}
m_{i}^{0}=m_{0}-\sum_{i=1}^{i-l}\left[\left(1+k_{l}\right) \beta_{l} \tau_{\imath}+m_{H, l}\right] \\
m_{i}^{f}=m_{0}-\sum_{l=1}^{i-1}\left[\left(1+k_{l}\right) \beta_{l} \tau_{l}+m_{H, l}\right]-\beta_{i} \tau_{i}
\end{array}\right\} \quad i=1, \ldots, N
$$

where $m_{O}$ is the lift-off mass. The summations in equations (19) (and all other similar summations) are defined to be zero whenever the lower summation limit exceeds the upper limit. The superscripts 0 and $f$ refer to conditions at the beginning and the end of each stage, respectively.

In order to calculate the final payload, the velocity impulse discussed earlier must first be considered. This velocity impulse is added after the desired orbit is achieved, with the use of the $\mathbb{N}^{\text {th }}$ stage for propulsion. After this maneuver is completed, the $\mathbb{N}^{\text {th }}$ stage hardware is jettisoned. The propellant required for the velocity impulse is

$$
\Delta m_{I}=m_{N}^{f}\left(1-e^{-\Delta V_{I} \beta_{N} / T_{N}}\right)
$$

By use of equation (19b), the final payload can be written

$$
m_{P L}=r\left\{m_{O}-\sum_{i=1}^{N-1}\left[\left(1+k_{\imath}\right) \beta_{\imath} \tau_{\imath}+m_{H, l}\right]-\beta_{N} \tau_{N}\right\}-k_{N} \beta_{N} \tau_{N}-m_{H, N}
$$

where $r$ has been defined as

$$
r=-k_{N}+\left(1+k_{N}\right) e^{-\Delta V_{I} \beta_{N} / T_{N}}
$$


The variations $d r_{i}^{0}$, $d m_{i}^{f}$, and $d m_{P L}$ can now be expressed in terms of the variations in burning times by differentiating equations (19) and (20).

$$
\begin{aligned}
& d m_{i}^{0}=-\sum_{i=1}^{i=1}\left(1+k_{l}\right) \beta_{l} d \tau_{l} \\
& \left.d m_{i}^{f}=-\sum_{i=1}^{i-1}\left(I+k_{l}\right) \beta_{l} d \tau_{l}-\beta_{i} d \tau_{i}\right\} \\
& i=1, \ldots, \mathbb{N} \\
& d m_{P L}=-r \sum_{l=1}^{N-1}\left(1+k_{\imath}\right) \beta_{\tau} d \tau_{\tau}-\left(r+k_{N}\right) \beta_{N N} d \tau_{N}
\end{aligned}
$$

By use of equations (22), the terms in equation (13) involving $d m_{i}$ can be simplified and expessed ir territs of the variations $d \tau_{i}$

$$
\begin{aligned}
\sum_{i=2}^{\mathbb{N}}\left(\lambda_{5} d m_{i}\right)_{t_{i-1}}^{t_{i}}= & \sum_{i=2}^{N-I} \lambda_{5}^{i_{k_{i}} \beta_{i}} d \tau_{i}-\lambda_{5}^{N} \sum_{i=1}^{N-I}\left(I+k_{i}\right) \beta_{i} d \tau_{i} \\
& -\lambda_{5}^{N} \beta_{N} d \tau_{N}+\lambda_{5}^{I}\left(I+k_{1}\right) \beta_{I} d \tau_{I}
\end{aligned}
$$

where

$$
\lambda_{5}^{i} \equiv\left(\lambda_{5}\right)_{t=t_{i}}
$$

Also, the time variation terrs in equation (13) can be expressed in terms of variations in the stage burning times:

$$
\sum_{i=2}^{\mathbb{N}}\left(c_{i} d t\right)_{t_{i-1}}^{t_{i}}=\sum_{i=2}^{\mathbb{N}} c_{i} d \tau_{i}
$$

The variation of $g$ is salculated by combining equations (2) and (22c):

$$
d g=r \sum_{1=1}^{N-1}\left(1+k_{1}\right) \beta_{1} d \tau_{1}+\left(r+k_{N}\right) \beta_{N} d \cdot \tau_{N}
$$

The variable $\lambda_{5}^{\mathbb{N}}$ can be eliminated from equation (23) by use of the following identity :

$$
\lambda_{5}^{\mathbb{N}} \equiv-\sum_{\substack{i=i+1 \\ \beta_{l} \neq 0}}^{\mathbb{N}}\left(\frac{i_{2}}{\beta_{l}}-\lambda_{5}^{l}\right)+\sum_{\substack{i=i+1 \\ \beta_{l} \neq 0}}^{\mathbb{N}}\left(\frac{C_{2}}{\beta_{2}}-\lambda_{5}^{i}-1\right)+\sum_{\substack{i=i+1 \\ \beta_{l}=0}}^{\mathbb{N}}\left(\lambda_{5}^{2}-\lambda_{5}^{l-1}\right)+\lambda_{5}^{i}
$$

It is convenient to define 


$$
\left.\begin{array}{ll}
S_{i}^{f}=\frac{C_{i}}{\beta_{i}}-\lambda_{5}^{i} & \beta_{i} \neq 0 \\
S_{i}^{O}=\frac{C_{i}}{\beta_{i}}-\lambda_{5}^{i-1} & \beta_{i} \neq 0 \\
S_{i}^{f}=0 & \beta_{i}=0 \\
S_{i}^{O}=0 & \beta_{i}=0
\end{array}\right\} \quad \begin{gathered}
i=2, \ldots ., \\
S_{1}^{f}=-\frac{1}{\beta_{1}}\left(\lambda_{1} \frac{\partial u}{\partial \tau_{1}}+r \lambda_{2} \frac{\partial \omega}{\partial \tau_{1}}+\lambda_{3} \frac{\partial r}{\partial \tau_{1}}+\lambda_{4} \frac{\partial \varphi}{\partial \tau_{1}}\right)
\end{gathered}
$$

A.lso, note that $\dot{\lambda}_{5}=0$ for coast phases, so that for $T_{i}=0$,

$$
\lambda_{5}^{i}=\lambda_{5}^{i-1}
$$

By use of equations (27) and (28), equation (26) becomes

$$
\lambda_{5}^{N}=-\sum_{i=i+1}^{N}\left(S_{l}^{f}-S_{l}^{O}\right)+\lambda_{5}^{i}
$$

Equations (16) to (18), (23) to (25) and (29) are now combined with equation (31) to give

$$
\begin{aligned}
d J & =\sum_{\substack{i=1 \\
\beta_{i} \neq 0}}^{N-I}\left\{\left(1+k_{i}\right) \beta_{i}\left[r+\sum_{i=i+1}^{N}\left(s_{i}^{f}-s_{i}^{0}\right)\right]+\beta_{i} s_{i}^{f}\right\} d \tau_{i} \\
& +\sum_{I=2}^{N} C_{i} d \tau_{i}+\left[\left(r+k_{N}\right) \beta_{N}+\beta_{N} S_{N}^{f}\right] d \tau_{N}-\left(\lambda_{i} \frac{\partial u}{\partial \alpha}+r \lambda_{2} \frac{\partial \omega}{\partial \alpha}+\lambda_{3} \frac{\partial r}{\partial \alpha}+\lambda_{4} \frac{\partial \varphi}{\partial \alpha}\right)_{t=t_{I}} d \alpha \\
& +\sum_{j=0}^{4}\left(\lambda_{I} \frac{\partial u}{\partial \eta_{j}}+r \lambda_{2} \frac{\partial \omega}{\partial \eta_{j}}+\lambda_{3} \frac{\partial r}{\partial \eta_{j}}+\lambda_{4} \frac{\partial \varphi}{\partial \eta_{j}}\right)_{t=t_{N}} d_{\eta_{j}}=0
\end{aligned}
$$

The variations in equation (30) are all independent, so that the form of equation (14) has been achieved, with

$$
\begin{aligned}
& G\left(\tau_{i}\right)=\left(1+k_{i}\right) \beta_{i}\left[\gamma+\sum_{i=1+1}^{N}\left(S_{i}^{f}-S_{l}^{0}\right)\right]+\beta_{i} S_{i}^{f}, \beta_{i} \neq 0 \\
& i=1, \ldots, N-I
\end{aligned}
$$




$$
\begin{aligned}
& G\left(\tau_{i}\right)=C_{i}, \beta_{i}=0 \quad i=2, . ., N-I \\
& G\left(\tau_{N}\right)=\left(r+k_{N}\right) \beta_{N}+\beta_{\mathbb{N}} S_{\mathbb{N}}^{f} \\
& G(\alpha)=-\left(\lambda_{I} \frac{\partial u}{\partial \alpha}+r \lambda_{2} \frac{\partial \omega}{\partial \alpha}+\lambda_{3} \frac{\partial r}{\partial \alpha}+\lambda_{4} \frac{\partial p}{\partial a}\right)_{t=t_{I}} \\
& G\left(\eta_{j}\right)=\left(\lambda_{1} \frac{\partial u}{\partial \eta_{j}}+r \lambda_{2} \frac{\partial \omega}{\partial \eta_{j}}+\lambda_{3} \frac{\partial r}{\partial \eta_{j}}+\lambda_{4} \frac{\partial Q}{\partial \eta_{j}}\right)_{t=t_{N}} \quad j=1, \ldots, 4
\end{aligned}
$$

From equations (11) and (27),

$$
\begin{aligned}
& \mathrm{s}_{i}^{\mathrm{f}}=\frac{\left[\left(\frac{\mu}{r^{2}}-\omega^{2} r\right) \lambda_{1}+2 u \omega \lambda_{2}-u \lambda_{3}-\omega \lambda_{4}-\frac{\mathrm{T}_{i}}{\mathrm{~m}_{i}^{\frac{f}{i}}} \sqrt{\lambda_{1}^{2}+\lambda_{2}^{2}}\right]_{t=t_{i}}}{\beta_{i}}
\end{aligned}
$$

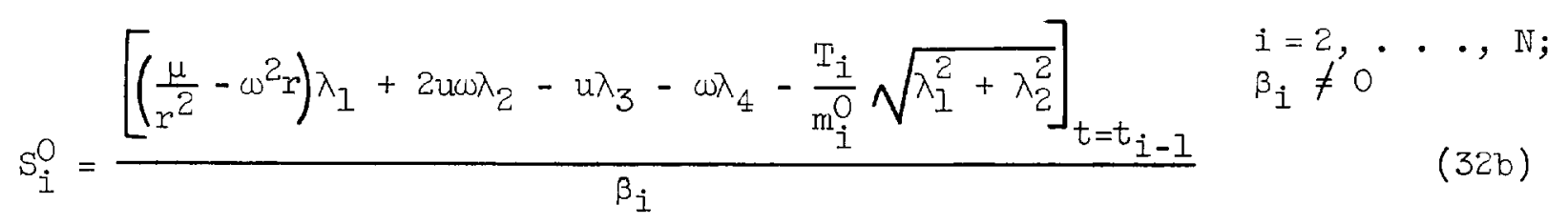

$$
\begin{aligned}
& C_{i}=\left[\left(\frac{\mu}{r^{2}}-\omega^{2} r\right) \lambda_{1}+2 \omega \omega \lambda_{2}-u \lambda_{3}-\omega \lambda_{4}\right]_{\beta_{i}=0 ; t_{i-1} \leq t \leq t_{i}}
\end{aligned}
$$

Since equations (32) do not contain $\mathrm{C}$ or $\lambda_{5}$, equation (7e) need not be integrated to evaluate equations (31), as indicated earlier.

\section{Boundary Value Problem}

The determination of an optimum trajestory requires the simultaneous integration of the equations of motion and the Euler-Lagrange equations. A set of initial conditions (state variables and Lagrange multipliers) and staging times is required in order to specify the trajectory uniquely.

The trajectory thus generated must satisfy $\mathbb{N}+5$ final conditions, corresponding to the $N+5$ independent problem variables in equation (30). For specified variables, the final conditions have the form

$$
x_{j}=x_{j, d}
$$

where the subscript $d$ indicates the desired final value. For unspecified variables, equations (31) supply auxiliary final conditions with the form 
$G\left(x_{j}\right)=0$

Some of equations (33) are easily satisfied; for example, a specified burning time for any stage can be achieved simply by terminating that stage at the proper time during the integration. An iteration is required in order to satisfy the nontrivial final conditions, and variable initial conditions (equal to the number of final conditions) must be available. For the present problem, the Lagrange multipliers $\left(\lambda_{i}, i=1, \ldots . .4\right)$, the burning times of all stages being optimized, and the booster kick angle (if it is being optimized) are available as variable initial conditions.

The size of the iteration loop can be reduced by using the fact that equations (7) are homogeneous in the $\lambda$ 's. This implies that the choice of any one $\lambda$ is arbitrary and serves only as a scale factor for the others. The value of this multiplier can be chosen to satisfy one of equations (3I).

The iteration size can be further reduced when $\lambda_{4}=0$ is a required final condition, which occurs when the travel angle is unspecified (ref. 8). Since $\lambda_{4}$ is a constant and is continuous across staging, this final condition can be satisfied at $t=t_{1} ; \lambda_{4}$ is thus removed from the iteration. Equation (3ld) can also be evaluated at $t=t_{1}$, and the booster kick angle optimization can be similarly removed from the iteration.

The burning times of optimized stages can sometimes be removed from the iteration, along with an equal number of equations (31). The principle involved is similar to that used in removing equation (3ld): If all variables in the equation $G\left(\tau_{\eta}\right)=0$ can be calculated at (or previous to) $t=t_{\eta}$, stage $l$ can be terminated (during stage $l$ ) whenever the optimizing equation is satisfied. The number of optimized stages which can be thus removed depends on the characteristics of the particular problem.

Consider first two powered stages $\tau_{\tau}$ and $\tau_{m}, \tau<m$, with $k_{m} \neq 0$. Equation (3la) must be set equal to zero for $i=\imath$ and $i=m$, and the two result- 
Ing equations are combined to give

$$
\frac{\mathrm{S}_{l}^{f}}{1+\mathrm{k}_{l}}+\sum_{i=l+1}^{\mathrm{m}-1}\left(\mathrm{~S}_{i}^{f}-\mathrm{S}_{i}^{O}\right)+\frac{\mathrm{k}_{\mathrm{m}}}{1+\mathrm{k}_{\mathrm{m}}} \mathrm{S}_{\mathrm{m}}^{\mathrm{f}}-\mathrm{S}_{\mathrm{m}}^{\mathrm{O}}=0
$$

Equation (3la) (for $i=i$ ) can be satisfied by the choice of one of the $\lambda^{\prime} s$. Equation (35) cortains terms which can all be calculated at, or prior to, stage $m$ cutoff, and this equation can be used to terminate stage $m$. Specifically, stige $m$ is terminated when

$$
S_{m}=S_{m}^{f}=\frac{I+k_{m}}{k_{m}}\left[S_{m_{2}}^{0}-\frac{S_{l}^{f}}{I+k_{l}}-\sum_{i=l+1}^{m-1}\left(S_{i}^{f}-S_{i}^{0}\right)\right]
$$

If $k_{m}=0$, the term in $S_{m}^{f}$ disappears from equation (35), and this equation can then be used to terminate stage $m-1$ :

$$
\begin{aligned}
& S_{m-1}^{f}-S_{m}^{O}=\sum_{i=\imath+1}^{m-1} S_{i}^{O}-\sum_{i=i+1}^{m-2} S_{i}^{f}-\frac{S_{l}^{f}}{1+k_{l}} \quad m>\imath+1 \\
& S_{m}^{O}-\frac{S_{l}^{f}}{I+k_{l}}=0 \quad m=\imath+1
\end{aligned}
$$

The terms on the left side of equations (37) are evaluated at stage $m$ - 1 cutoff, and the terms on the right side are evaluated during previous stages. Fon the special case $m=\mathbb{N}, k_{\mathbb{N}} \neq 0$, equation (3lc) must be set equal to $z \in$ r.o. Equations (3la) (for $1=\imath$ ) and (3lc) are combined to give

$$
S_{\mathrm{N}}^{f}=-\frac{r+k_{N}}{k_{N}}\left[\frac{s_{l}^{f}}{1+k_{l}}-S_{N}^{O}+\sum_{i=l+1}^{N-1}\left(s_{i}^{f}-s_{i}^{0}\right)\right]
$$

Equation (38) is used to terminate stage $\mathbb{N}$.

For coast phases to be optimized, equations (31) supply criteria for termiration of the prerious stage, similar to equation (37). For such cases, stage ? - I is terminated when $\mathrm{C}_{2}=0$, where stage $l$ is the coasting stage to be optinized. 


\section{RESULTS}

In order to demonstrate the validity of the equations and the feasibility of the variational technique, parametric results are presented and compared with the overall optimum solutions obtained by using the variational technique. Two- and three-stage launch vehicles were optimized by use of the equations developed. The results presented include a two-stage vehicle flown to circular orbit and a three-stage vehicle flown to Earth escape through a circular parking orbit. In these results, both fixed and variable hardware weights were used, and the propellant loadings and booster kick angle have been optimized. Parametric results are also presented, which show the variation of payload with propellant loadings and kick angle.

\section{Vehicle Definition}

The vehicle chosen for this study is a hypothetical three-stage launch vehicle consisting of two chemical stages and one nuclear stage. The assumptions on propulsion and weights are listed in table I.

TABLE I. - LAUNNCH VEHICLE PROPULSION AND WEIGHT DATA

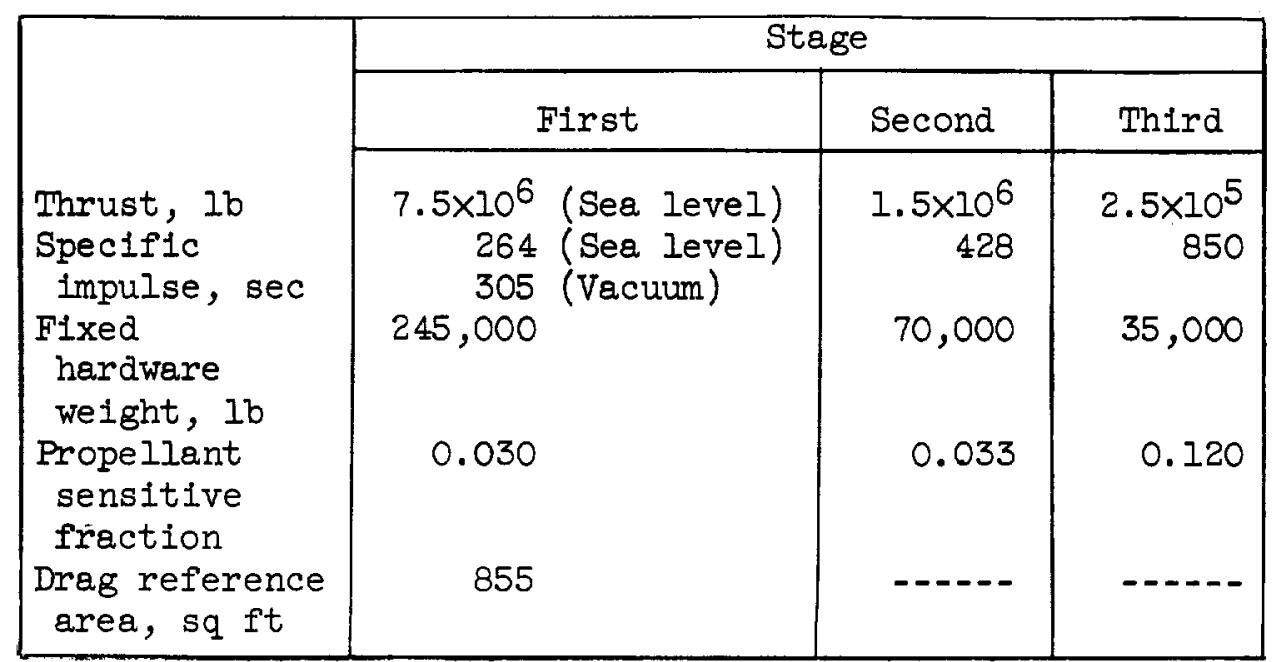

The first stage is based on the Saturn SI-C stage, consisting of five F-I engines using RP-LOX propellants. The engine performance data are used for illustrative purposes only and are not necessarily consistent with present 
F-? ralis:. Tre secord stage consists of one M-I engine using liquid hydrogen and Itquid oxygen as propellants. The third stage is a nuclear stage. The jaunch thrust-to-weight ratio was fixed at 1.25 for this study, and the launch azimuth was $90^{\circ}$.

\section{Numerical Results}

A typical propeliart tank sizing study was conducted with the vehicle defined in table I. Iri this study, the stages are assumed to have variable tank s...ze, and the optiniu propellant capacities are determined by flying possible mission:s of irterest.

Whe fins mistor is fiown to Eanth escape energy with three stages. This riesion is typical of linar and planetiary probes and orbiters. A parking orbit ascerit mode is used, wherein all three stages are used to enter a parking orbit. of a 121 natical-mile altitude (similar to the Apolio mission), after which the third stage is burred to escape. Fhe second burning of the third stage is assired to be impuisire, with a velocity inerement of 10563 feet per second. The maximur payload capatility for tinis mission is obtained by optimizing the booster kick argle as well as the propelant loadings of the three stages. By use of the equations derived eariier, maximum payload can be obtained with a single (correnged) solution, represented by the optimum point in figure 2 .

With the parametmis procedure, the maximum payload is obtained as the en:lope of payioad points with all possible combinations of propellant load$\therefore$ rgs ara booster kizk angle. The increased effort (rumber of solutions) rewinced for this proedure sis obrious. In addition to the parametric curves shown in figire 2, each poirt on the curves had to be obtained at an optimum i... angle, which required an additional family of curves (not shown in the Ifgure), with kick angle as the independert parameter. As can be seen from f...e 2, the payload capability and optimized propellant loading obtained from the variational provedure agree with the parametric results. 
Another mission of interest is two stages flown to a circular orbit, with the use of the first and second stages from table I. Results for this mission are presented in figures 3 and 4. In figure 3, with the use of the parametric procedure, payload capability is presented as a function of first-stage propellant loading for various booster kick-angles. The maximum payload for each kick angle is determined from the figure and presented as a function of kick angle in figure 4. The case flown by use of the variational technique is represented by the optimum point in figure 4 , and the payload obtained is in agreement with the envelope, as before.

Frequently, the propellant tanks of the various stages are sized for one mission and fixed at these values for all other missions. Propellant loadings can still be optimized in such cases but with the restriction that the propellant loadings cannot exceed the propellant capacities of the tanks.

In figure 5, the three-stage Earth escape mission is reoptimized with fixed tanks for the first and second stages. The tank weights and propellant capacities used are based on optimum values for the two-stage orbit mission. Since the maximum propellant capacities of the first and second stages were not exceeded in the optimum case, the propellant loadings of these stages were offloaded to the optimum values. The parametric results and the variational point are compared (as in fig. 2), and the results are in agreement.

CONCLUDING REMARKS

A technique is presented which allows simultaneous optimization of the thrust direction profile and vehicle control parameters for multistage launch vehicles. The agreement of parametrlc and optimum results presented in figures 2 to 5 demonstrates the correctness of the optimizing equations.

The amount of effort (and computer time) saved by the variational technique in determining the maximum payload capability can be seen by referring to the figures. In figure 2, for example, approximately 80 parametric data points are 
required to optimize the three-stage propellant loadings and booster kick angle (not shown in the figure). When good initial guesses are available, the computer time required to obtain the overall optimum solution is about the same as for each parametric solution. The time saving is therefore proportional to the number of parametric cases required for complete optimization.

\section{APPENDIX A}

SYMBOLS

C constant of integration

E Weierstrass excess function

e eccentricity

F $\quad \sum_{j=1}^{5} \lambda_{j} f_{j}$

$f$ constraint equation

G $\partial J / \partial x$

$g$ function of initial and final conditions to be minimized

$\mathrm{J}$ functional to be minimized by variational methods

$\mathrm{k}$ propellant sensitive mass fraction

m mass, slugs

$r$ radius, ft

$S$ functions defined in eqs. (27)

$\mathrm{T}$ thrust, $\mathrm{Ib}$

$t$ time, sec

u radial velocity, ft/sec

$v$ velocity, ft/sec

$\mathrm{x}$ problem variable

a booster kick angle, rad

$\beta$ mass flow rate, slug/sec

$\gamma$ function defined in eq. (21)

$\eta \quad$ generalized state variable

$\lambda$ Lagrange multiplier 
$\mu \quad$ Earth force constant, cu ft/ $/ \mathrm{sec}^{2}$

$\tau$ burning time, sec

$\varphi$ polar angle, rad

$\psi \quad$ thrust direction, rad

$\omega$ angular velocity, rad/sec

Subscripts :

d desired value

$\mathrm{H}$ fixed hardware

I impulsive

i stage number

j variable number

$\mathrm{k} \quad$ variable number

$2 \quad$ stage number

N last stage

PL payload

p propellant

s structure

0 initial

Superscripts :

$f$ end of stage

1 refers to $t=t_{1}$

0 beginning of stage

- derivative with respect to time

$+\quad$ after staging

- before staging

$\rightarrow \quad$ vector 


\section{REF'ERENCES}

1. Goldsmith, M.: On the Optimization of Two-Stage Rockets. Jet Prop., vol. 27, no. 4, Apr. 1957, pp. 415-416.

2. Schurmann, Ernest E. H.: Optimum Staging Technique for Multistaged Rocket Vehicles. Jet Prop., vol. 27, no. 8, Aug. 1957, pp. 863-865.

3. Subotowicz, M.: The Optimization of the N-Step Rocket with Different Construction Parameters and propellant Specific Impulses in Each Stage. Jet Prop., vol. 28, no. 7, July 1958, pp. 460-463.

4. Hall, H. H.; and Zambelli, E. D.: On the Optimization of Multistage Rockets. Jet Prop., vol. 28, no. 7, July 1958, pp. 463-465.

5. Weisbord, L.: Optimum Staging Techniques. Jet Prop., vol. 29, no. 6, June 1959, pp. 445-446.

6. Cobb, Edgar R.: Optimum Staging Technique to Maximize Payload Total Energy. ARS J., vol. 31, no. 3, Mar. 1961, pp. 342-344.

7. Coleman, John J.: Optimum Stage-Weight Distribution of Multistage Rockets. ARS J., vol. 31, no. 2, Feb. 1961, pp. 259-261.

8. Zimmerman, Arthur V.; MacKay, John S.; and Rossa, Leonard G.: Optimum LowAcceleration Trajectories for Interplanetary Transfers. NASA TN D-1456, 1963.

9. Melbourne, William G.; and Seuer, Carl G., Jr.: Optimum Thrust Programs for Power-IImited Propulsion Systems. Rept. No. TR 32-118, Jet Prop. Lab., C.I.T., June 15, 1961.

10. MacKay, John S.; and Rossa, Leonard G.: A Variational Method for the Opt1mization of Interplanetary Round-Trip Trajectories. NASA TN D-1660, 1963.

11. Jurovics, Stephen: Optimum Steering Program for the Entry of a Multistage Vehicle into a Circular orbit. ARS J., vol. 31, no. 4, Apr. 1961, pp. $518-522$. 
12. Mason, J. D.; Dickerson, W. D.; and Smith, D. B.: A Variational Method for Optimal Staging. Paper presented at AIAA 2nd Aerospace Sciences Meeting, Paper No. 65-62, AIAA, Jan. 1965.

13. Denbow, C. H.: Generalized Form of the Problem of Bolza. Ph.D. Thesis, Univ. of Chicago, 1937.

14. Hunt, R. W.; and Andrus, J. F.: Optimization of Trajectories having Discontinuous State Varaibles and Intermediate Boundary Conditions. Paper presented at SIAM Meeting, Monterey (Calif.), Jan. 1964.

15. Stancil, R. T.; and Kulakowski, I. J.: Rocket Boost Vehicle Mission Optimization. ARS J., vol. 31, no. 7, July 1961, pp. 935-942.

16. Teren, Fred; and Spurlock, Omer F.: Payload Optimization of Multistage Launch Vehicles. Proposed NASA TN.

17. Bliss, G. A.: Lectures on the Calculus of Varlations. Univ. Chicago Press, 1946.

18. Leitmann, G.: On a Class of Variational Problems in Rocket Flight. J. Aero/Space Sci., vol. 26, no. 9, Sept. 1959, pp. 586-591. 


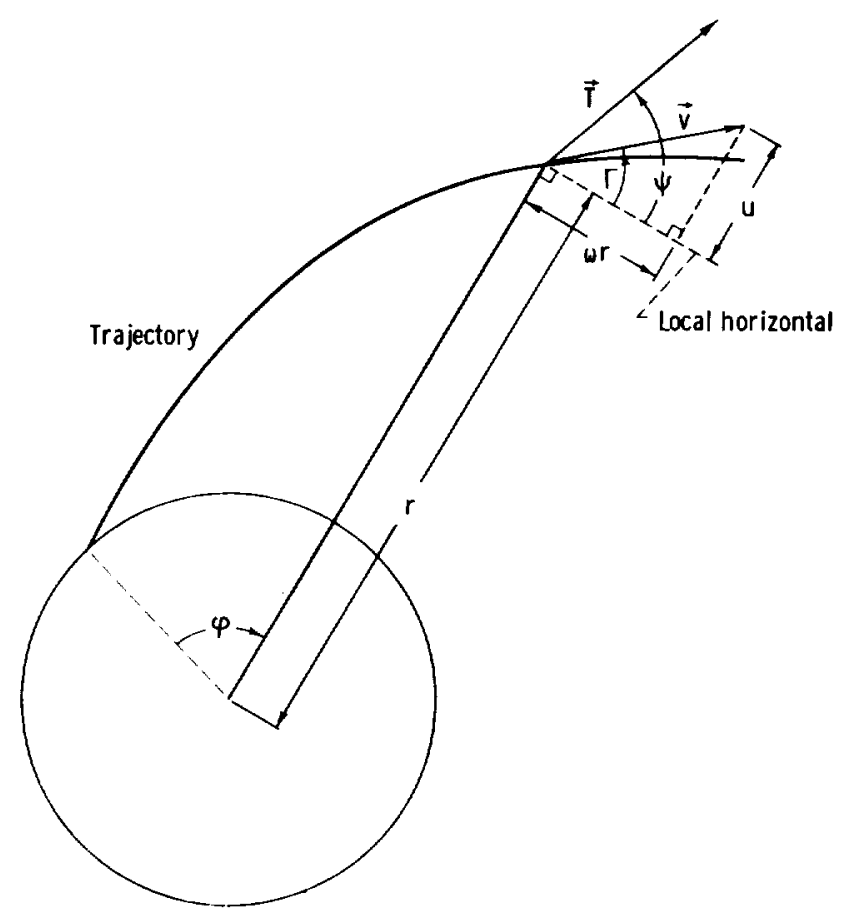

Figure 1. - Definition of problem variables.

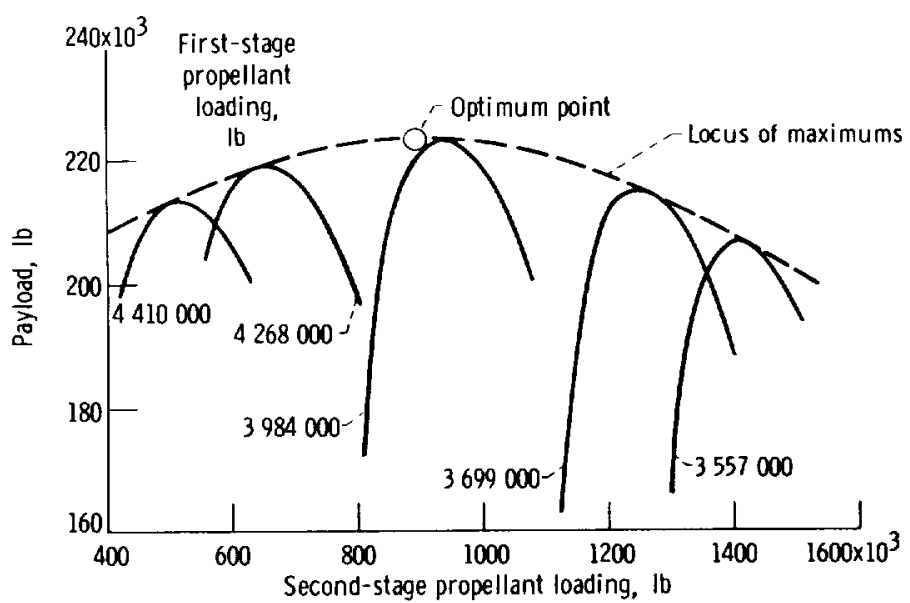

Figure 2. - Payload capability as function of second-stage propellant loading. Three stages to Earth escape via 121 -nautical-mile parking orbit; variable tanks; first-stage propellant loading at optimum point, 4028000 pounds; - third-stage propellant loading at optimum point, 317000 pounds. 


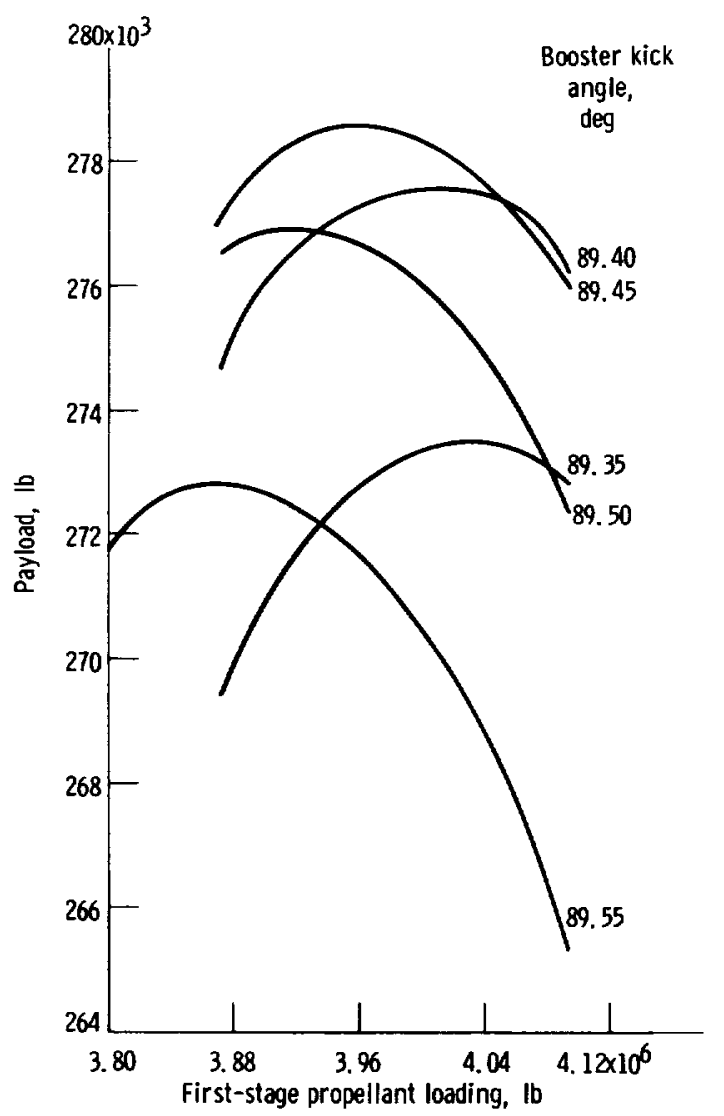

Figure 3. - Payload capability as function of first-stage propellant loading. Two stages to 121-nautical-mile orbit. 


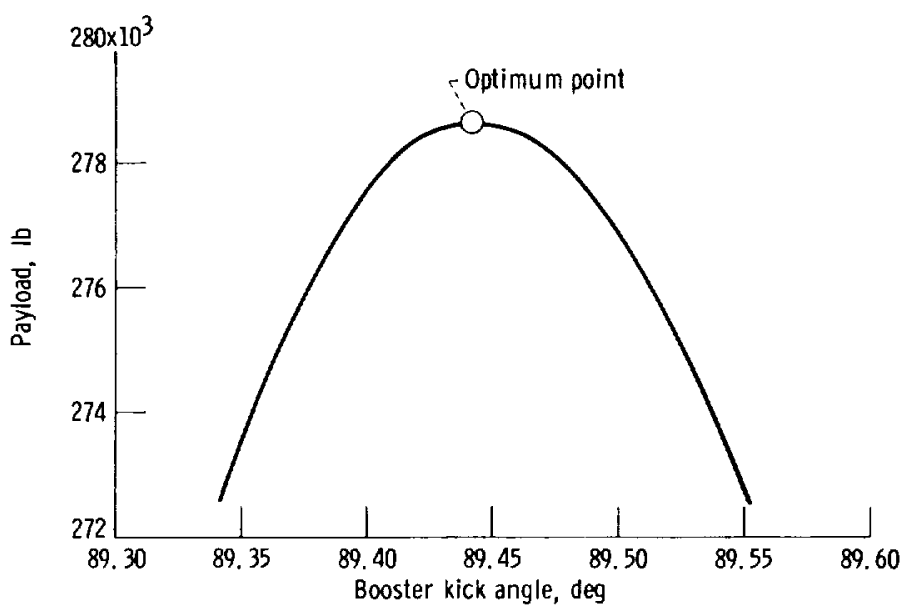

Figure 4. - Payload capability as function of booster kick angle. Propellant loadings optimized; two stages to 121-nautical-mile orbit; first-stage propellant loading at optimum point, 3980000 pounds; second-stage propellant loading at optimum point, 1276000 pounds.

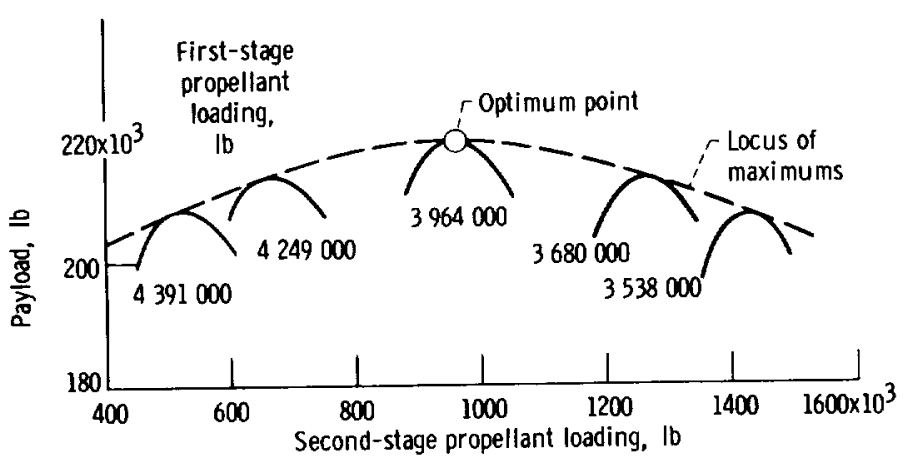

Figure 5. - Payload capability as function of second-stage propellant loading. Three stages to Earth escape via 121 -nautical-mile parking orbit; fixed tanks; first-stage propellant loading at optimum point, 3964000 pounds; third-stage propellant loading at optimum point, 310000 pounds. 
